\title{
Roadmap to eliminate gastric cancer with Helicobacter pylori eradication and consecutive surveillance in Japan
}

\author{
Masahiro Asaka - Mototsugu Kato • \\ Naoya Sakamoto
}

Received: 2 October 2013/Accepted: 2 October 2013/Published online: 27 October 2013

(C) The Author(s) 2013. This article is published with open access at Springerlink.com

\begin{abstract}
In Japan, the annual number of deaths from gastric cancer is approximately 50,000 and there has been no change over the last 50 years. So far, all efforts have been directed toward improving the detection of early gastric cancer by barium X-ray and endoscopy, since early cancer has a good prognosis, resulting in Japan having the best diagnostic capability for early gastric cancer worldwide. The 5-year survival rate of gastric cancer patients exceeds $60 \%$ in Japan and is much higher than that in Europe and the US (20\%) because of this superior diagnosis of early gastric cancer. In February 2013, national health insurance coverage for Helicobacter pylori eradication therapy to treat $H$. pylori-associated chronic gastritis became available in Japan. H. pylori-associated gastritis leads to development of gastric and duodenal ulcers and gastric polyps. Therefore, providing treatment for gastritis is likely to substantially decrease the prevalence of both gastric and duodenal ulcers and polyps. Because treatment for $H$. pylori-associated gastritis, which leads to atrophic gastritis and gastric cancer, is now covered by health insurance in Japan, a strategy to eliminate gastric cancerrelated deaths by taking advantage of this innovation was planned. According to this strategy, patients with gastritis
\end{abstract}

M. Asaka ( $\square)$

Cancer Preventive Medicine, Hokkaido University Graduate

School of Medicine, Kita 12, Nishi 7, Kita-ku,

Sapporo 060-0812, Japan

e-mail: maasaka@med.hokudai.ac.jp

M. Kato

Division of Endoscopy, Hokkaido University Hospital,

Sapporo, Japan

N. Sakamoto

Department of Gastroenterology, Hokkaido University Graduate

School of Medicine, Sapporo, Japan will be investigated for $H$. pylori infection and those who are positive will receive eradication therapy followed by periodic surveillance. If this strategy is implemented, deaths from gastric cancer in Japan will decrease dramatically after $10-20$ years.

Keywords Gastric cancer - Helicobacter pylori . Elimination of gastric cancer - Eradication of Helicobacter pylori

\section{Introduction}

After the discovery of Helicobacter pylori (H. pylori) in 1983 [1], the causal relationship between this bacterium and gastritis and/or gastric cancer has been steadily elucidated. In 1994, H. pylori was classified as a definite carcinogen by the International Agency for Research on Cancer (IARC) of the World Health Organization (WHO) [2]. Subsequently, many clinical studies were conducted in various countries to determine whether eradication of H. pylori could contribute to the prevention of gastric cancer. However, the very low incidence of gastric cancer among the subjects meant that sufficient data for statistical analysis were not obtained. In 2008, randomized multicenter clinical study conducted in Japan revealed that eradication of $H$. pylori reduced the incidence of secondary gastric cancer by about two-thirds after endoscopic mucosal resection (EMR) of early gastric cancer [3], suggesting the usefulness of $H$. pylori eradication for prevention of gastric cancer. However, this study also showed that $H$. pylori eradication did not completely eliminate gastric cancer. Therefore, to eliminate gastric cancer, periodical surveillance would be required after $H$. pylori eradication. Thus, to achieve the elimination of gastric cancer in Japan, the 
important issue is how to combine primary prevention through $H$. pylori eradication with secondary prevention through surveillance. Fortunately, the Ministry of Health, Labour and Welfare of Japan (MHLW) approved national health insurance coverage for eradication therapy in patients with gastritis caused by $H$. pylori infection (chronic active gastritis) on February 21 in 2013 for the first time in the world. Here, we present a roadmap for the elimination of gastric cancer in Japan, focusing on eradication of H. pylori.

Current status and characteristics of screening for gastric cancer in Japan

Approximately $60 \%$ of gastric cancer patients worldwide are found in only three East Asian countries (Japan, China, and Korea), and the disease seems to be endemic to this area [4]. Gastric cancer was the most common cause of cancer death in Japan until it was replaced by lung cancer in 1995 [5]. Thanks to concerted efforts by clinical and fundamental researchers, the concept of early gastric cancer was proposed in Japan in 1963. At that time, early gastric cancer was defined as a lesion with infiltration of tumor cells limited to the mucosa or submucosa, irrespective of lymph node metastasis $[6,7]$.

The prognosis of early gastric cancer is far better than that of advanced cancer, with a 5-year survival rate exceeding $90 \%$ [8]. Therefore, many studies in Japan have focused on how to diagnose effectively early gastric cancer. As a result, early cancer now accounts for nearly $60 \%$ of all gastric cancers detected in Japan. This has not been reported in any other country and suggests high diagnostic capability for early cancer in the country. The survival rate of gastric cancer patients depends on the stage of their tumor. It exceeds $90 \%$ for stage I (including early gastric cancer), while it is under $20 \%$ for stage IV. Thus, a higher stage means a worse prognosis [9], hence it is important to detect as many early cancers as possible for improvement of gastric cancer mortality. The efforts made so far have led to an overall 5-year survival rate of better than $65 \%$ for gastric cancer patients in Japan [9]. In other countries, including the US and Europe, the 5-year survival rate of gastric cancer patients is reported to be only 10-25\% (Fig. 1) [10-12]. This is not because treatment of gastric cancer is superior in Japan to that in other countries, but because the detection rate of early cancer is much lower outside Japan. In other words, the stage distribution of gastric cancer patients receiving treatment in Japan is likely to be different from that in other countries where there is little emphasis on detecting early gastric cancer. In the US and Europe, intramucosal carcinoma is not even considered to be cancer and is classified as dysplasia [13]. Thus, researchers in the US and Europe have speculated that the

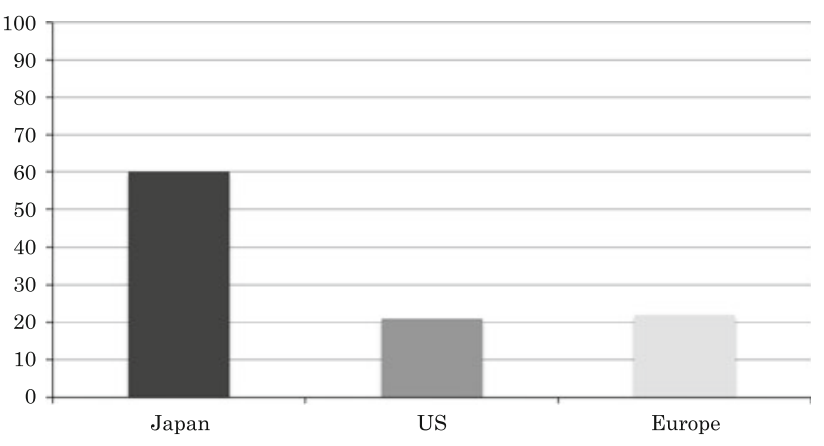

Fig. 1 Five-year survival rate in Japan, US and Europe (\%)

diagnosis and treatment of precancerous lesions as early gastric cancer improves the prognosis in Japan compared with other countries. There is undoubtedly a difference of diagnostic criteria between Japan and the US/Europe. While Japanese pathologists make a diagnosis of gastric cancer based on the presence of atypical nuclei in gastric mucosal cells and atypical glandular or ductal structures, pathologists in the US and Europe will diagnose gastric dysplasia instead of gastric cancer when atypical glandular and ductal structures do not extend beyond the muscularis mucosa [13]. This difference in the diagnostic criteria for gastric cancer between Japan and the US/Europe is an issue that seems to be difficult to resolve [14]. However, based on the findings that $30-60 \%$ of lesions diagnosed as dysplasia show progression to gastric cancer within a few years [15-17] and that examination of larger biopsy specimens leads to diagnosis of more lesions as cancer [18], high-grade dysplasia should be classified as intramucosal gastric cancer and be treated aggressively in order to improve the prognosis of gastric cancer.

In Japan, endoscopic surgery is commonly performed for intramucosal gastric cancer, a form of early gastric cancer, while such treatment is uncommon in Europe and the US where intramucosal lesions are not regarded as cancerous. Even if physicians in Europe and the US accept the concept of early gastric cancer, they need to develop techniques for diagnosis and endoscopic surgery [17]. Possibly because of inadequate diagnostic techniques for early gastric cancer, the prognosis of gastric cancer is poor in other countries. The skill of Japanese physicians with regard to early diagnosis and endoscopic treatment of gastric cancer is unsurpassed and contributes greatly to the management of patients with this cancer in Japan. To achieve improvement of the prognosis of gastric cancer in other countries, it would be necessary for the concept of early gastric cancer to be accepted and the same level of technical skill in the management of early gastric cancer as in Japan to be established, which could lead to the promotion of gastric cancer elimination by combining H. pylori eradication therapy with surveillance. 
Prevention of gastric cancer by eradication of $H$. pylori

As it has become clear that $H$. pylori infection is an important risk factor for gastric cancer, the issue of whether $H$. pylori eradication therapy can decrease the incidence of gastric cancer has attracted increasing attention. Intervention studies to assess the preventative effect of H. pylori eradication on gastric cancer have been conducted in healthy individuals worldwide. However, the incidence of gastric cancer is very low in the US and Europe, and the study populations were not large enough to detect a significant effect of eradication therapy, resulting in the discontinuation of most studies [19].

In 2004, a report on a large intervention study performed in China was published [20]. This study involved 1,630 H. pylori-infected residents of Fujian province in China where the mortality rate due to gastric cancer is high. During the follow-up period, gastric cancer developed in 7 and 11 subjects from the eradication and placebo groups, respectively, and there was no significant difference between the two groups $(p=0.33)$. However, among patients without precancerous lesions (atrophy, intestinal metaplasia, or dysplasia), there were no cases of gastric cancer in the $H$. pylori eradication group and there was a significantly lower incidence of gastric cancer compared with the placebo group $(n=6)(p=0.02)$. Interestingly, all of the cancers diagnosed after $H$. pylori eradication were advanced and there were no cases of early gastric cancer, which was a surprising result in view of the situation in Japan where at least $60 \%$ of gastric cancers are diagnosed at an early stage. Such an outcome suggests that the endoscopists involved in this study might not be skilled in the diagnosis of early gastric cancer. Accordingly, the patients who were classified as having atrophic gastritis or intestinal metaplasia may well have included many patients with early gastric cancer. $H$. pylori infection is strongly associated with early gastric cancer rather than advanced cancer [21], suggesting that investigation of the prevention of gastric cancer by $H$. pylori eradication therapy is problematic outside Japan because early gastric cancer is unlikely to be diagnosed.

Assessment of the design of a new prospective study on the basis of previous studies indicated that a clinical trial with a small sample size and short follow-up period should enroll patients with early gastric cancer who have undergone EMR, since they represent the population most likely to develop advanced gastric cancer. The annual incidence of gastric cancer has been reported to be only $0.1-0.4 \%$ in H. pylori-positive patients with atrophic gastritis [22, 23], while the annual incidence of metachronous recurrence is far higher $(3-5 \%)$ in patients who have undergone endoscopic surgery for early gastric cancer [24, 25]. We investigated the metachronous recurrence of gastric cancer in 544 patients who had undergone endoscopic treatment for early gastric cancer. They were randomly allocated to $H$. pylori eradication or non-eradication groups and were followed up by annual endoscopic examination for 3 years. As a result, metachronous recurrence was detected in 9 and 24 subjects from the eradication group and the non-eradication group, respectively, and the former had a significantly lower relapse rate ( $p<0.01$ according to intention-to-treat analysis) [3]. This prospective study had an adequate sample size to provide a definitive answer to the long controversial issue of whether gastric cancer could be prevented through $H$. pylori eradication. It demonstrated that $H$. pylori eradication therapy reduced the incidence of intestinal type gastric cancer by at least two-thirds and this effect was noted irrespective of whether patients had atrophic gastritis, intestinal metaplasia, or early gastric cancer. Thus, it was confirmed that most gastric cancer is associated with H. pylori infection and that the disease can be effectively prevented by eradication of this microorganism.

In 2009, a large-scale cohort study was reported, in which about 80,000 patients in Taiwan were followed up for 10 years after $H$. pylori eradication therapy [26]. The patients were assigned to an early eradication group (H. pylori eradication therapy was provided at the time of diagnosis) or a late eradication group (eradication therapy was performed at 1 year or more after diagnosis). It was found that the incidence of gastric cancer was markedly lower in the early eradication group than in the late eradication group. This study is important because it demonstrates that $H$. pylori eradication therapy inhibits the development of gastric cancer and that earlier eradication is more effective.

In 2006, You et al. [27] reported a study of 3,365 patients in China who were randomized to an $H$. pylori eradication group, a garlic group, or a vitamin group and were followed for 7.3 years, with the result being no inhibition of gastric cancer in any of the groups. However, longer follow-up for 15 years subsequently revealed significant inhibition of gastric cancer in the $H$. pylori eradication group (odds ratio, $0.61 ; p=0.032$ ) [28]. Although there was no significant preventive effect on gastric cancer of eradication therapy in the original randomized study, longer observation revealed a significant effect.

The above-mentioned three studies performed in East Asia provide strong evidence that $H$. pylori eradication therapy is effective for inhibiting gastric cancer. In Japan, Maehata et al. investigated the long-term clinical outcome following $H$. pylori eradication therapy and whether it prevented metachronous gastric cancer. They reported that eradication therapy inhibited the development of metachronous gastric cancer for 5 years, but there was no significant difference after longer follow-up [29]. However, the mean observation period of this study was only 3 years and 
the 10-year prognosis was assessed in very few patients, leading to lack of reliability. That is, the findings about the short-term prognosis may well be accurate, but no conclusion can be drawn regarding the long-term outcome.

After the JGSG study was completed and data obtained at $8-10$ years were analyzed, it was found that there was still a difference in the incidence of metachronous gastric cancer between the $H$. pylori eradication and non-eradication groups [30]. This indicates that the preventive effect of eradication therapy on gastric cancer persists for a long time.

Health insurance coverage for $H$. pylori eradication therapy in Japan

Cancers are classified into two broad categories, which are lifestyle-related and infection-related cancers. In the US and Europe, cancers related to infection account for a low percentage (10\% or less) of all cancers [31, 32]. In Japan, however, it has become clear that infection-related cancers account for approximately $25 \%$, including liver cancer caused by hepatitis viruses, cervical cancer due to papillomavirus, and gastric cancer related to $H$. pylori. Although cervical cancer is uncommon and accounts for a low percentage $(1.3 \%)$ of all cancers, gastric cancer and liver cancer account for about 17 and $6.5 \%$, respectively, and the total for these three cancers is nearly $25 \%$ [33]. Since it has become clear that most gastric cancer is due to H. pylori infection rather than lifestyle factors, it is time for major revision of the preventative strategies for gastric cancer. When it is suspected that a cancer is caused by infection, proactive preventative measures are likely to lead to a dramatic decrease in the incidence of that cancer, resulting in a significant decrease of cancer mortality. In Japan, preventative measures for liver cancer have been focused on hepatitis virus infection since 2002, leading to a reduction of mortality [34, 35]. However, the annual number of deaths from gastric cancer has remained at around 50,000 for the last few decades [36], suggesting that the current preventative measures are inadequate (Fig. 2). Even though there is a difference in the causative agent between liver cancer (viruses) and gastric cancer (a bacterium), preventive measures for gastric cancer should not be completely different from those for liver cancer. Thus, the fundamental measures for preventing gastric cancer should be shifted from conventional secondary prevention based on barium X-ray screening to primary prevention focused on $H$. pylori eradication therapy.

In 2009, the Japanese Society for Helicobacter Research published a guideline in which it is recommended that all $H$. pylori-infected people receive bacterial eradication therapy [37]. In response to this, the MHLW approved the extension of national health insurance coverage to $\mathrm{H}$. pylori

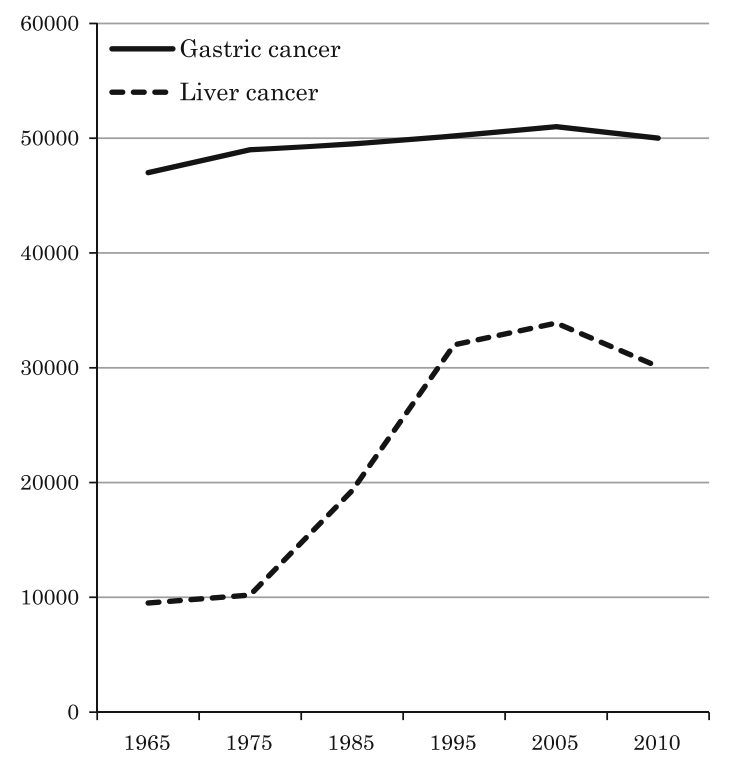

Fig. 2 Changes of deaths of gastric cancer and liver cancer in Japan

eradication therapy for three indications [i.e., patients with gastric mucosa-associated lymphoid tissue (MALT) lymphoma, patients who have undergone endoscopic surgery for early gastric cancer, and patients with idiopathic thrombocytopenic purpura (ITP)], in addition to patients with gastric and duodenal ulcer. This was the first time in the world that insurance coverage has been provided for H. pylori eradication therapy for indications other than gastric and duodenal ulcer and represents an innovative approach. Regarding the potential expansion of health insurance coverage for eradication therapy to include patients with chronic gastritis, the Japanese Society of Gastroenterology, the Japan Gastroenterological Endoscopy Society, and the Japanese Society for Helicobacter Research submitted a joint petition to the Minister of the MHLW. This public knowledge-based application led to the inclusion of $H$. pylori eradication therapy for patients with chronic gastritis on February 21, 2013. The MHLW notification states that eradication therapy is covered by the national health insurance scheme when a patient with endoscopically diagnosed chronic gastritis is positive for $\mathrm{H}$. pylori.

Within a few months of being infected, gastritis with neutrophil and lymphocyte infiltration develops in almost $100 \%$ of patients who have $H$. pylori infection. Such gastritis is called chronic active gastritis and is said to be specific to $H$. pylori infection [38]. Gastritis caused by H. pylori develops as a biological defense to the infection, resulting in the production of inflammatory cytokines such as IL-1 and IL-8 and the expansion of gastric mucosal inflammation [39]. Persistent inflammation gradually increases the fragility of the gastric mucosa and $H$. pyloriassociated gastritis progresses to atrophic gastritis over time. It has been demonstrated that progression takes 


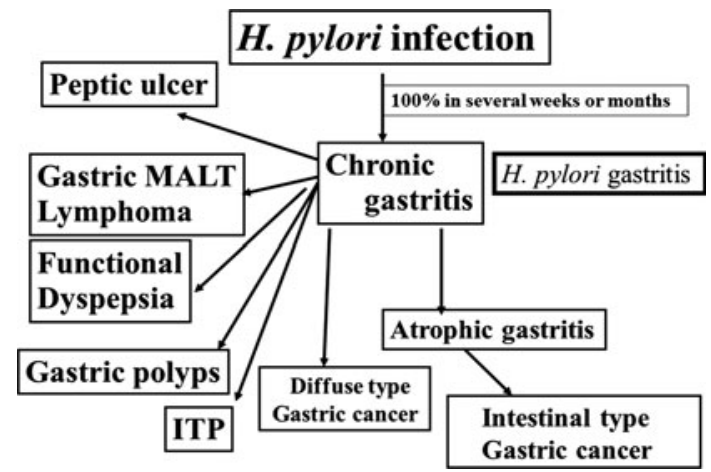

Fig. 3 Progress of $H$. pylori infection

10-20 years in about $80 \%$ of Japanese patients [40], and some cases of atrophic gastritis then progress to intestinal type gastric cancer. Correa's hypothesis has been widely accepted, which states that gastritis progresses to intestinal metaplasia, dysplasia, and then gastric cancer [41], and it has been virtually proved by the discovery of $\mathrm{H}$. pylori. The effects of gastric acid and stress on a background of H. pylori-associated gastritis can lead to the development of peptic ulcer. In contrast, gastritis that is not associated with $H$. pylori usually does not progress to ulceration even when stress occurs. It has become obvious that $H$. pylori-associated gastritis is also closely associated with gastric MALT lymphoma, functional dyspepsia (FD), hyperplastic gastric polyps, idiopathic thrombocytopenic purpura (ITP) and diffuse type gastric cancer (Fig. 3) [42,43] Thus, H. pylori-associated gastritis is the underlying cause of almost all gastric diseases, hence treatment of this gastritis through bacterial eradication therapy is likely to prevent most gastric conditions, including gastric cancer.

Kodama et al. observed the 10-year course by obtaining biopsy specimens from 5 different sites of the gastric mucosa, and found that the activity score and inflammation improved immediately after $H$. pylori eradication therapy and then remained stable for the next 10 years. They reported that atrophic change took longer to improve leukocytic infiltration, but nearly $80 \%$ of the changes had resolved after 10 years [44]. Their findings revealed that eradication therapy improved atrophic changes of $H$. pylori-associated gastritis, as well as leukocytic infiltration, indicating that this therapy is useful for the prevention of gastric cancer.

Strategy and roadmap for the elimination of gastric cancer in Japan

In order to eliminate gastric cancer in Japan, the strategy for adolescents should be different from that for elderly persons. This is because bacterial eradication in adolescents achieves nearly $100 \%$ prevention of gastric cancer, but the incidence of this cancer increases with advancing age $[43,45]$. We recommend a test-and-treat approach as the strategy for adolescents, which includes H. pylori testing of junior high school and high school students, followed by immediate $H$. pylori eradication therapy for those with a positive result. Eradication in adolescents should be able to prevent $H$. pylori-related diseases such as gastric ulcer and gastric polyps, as well as preventing the development of nearly $100 \%$ of gastric cancers. It is estimated that approximately $5 \%$ of all teenagers in Japan are positive for $H$. pylori [46], suggesting that the cost of this approach would not be so high. Some local governments have already scheduled free $H$. pylori testing for junior high school students.

The recent expansion of health insurance coverage allows individuals with symptoms such as gastric heaviness to present to hospital for the diagnosis and treatment of H. pylori-associated gastritis. To obtain health insurance coverage, endoscopy must be performed first for the diagnosis of gastritis, and most patients seem to have chronic gastritis by the time they undergo endoscopy. We expect that many patients with gastric cancer will be discovered during this endoscopic examination. This project thus includes a form of endoscopic screening supported by medical insurance. All patients in whom gastritis is diagnosed are supposed to receive $H$. pylori eradication therapy. In patients with obvious atrophic gastritis, periodic endoscopic follow-up is recommended every 1 or 2 years even after eradication therapy, while patients with no or mild atrophy and those who are negative for $H$. pylori infection can be followed by optional screening (such as personal medical check-up) instead of strategic screening (Fig. 4).

In Japan, the success rate of $H$. pylori eradication therapy is decreasing every year because of the increase in bacteria resistant to clarithromycin, but secondary eradication therapy with metronidazole achieves a high success rate $(>95 \%)$. That may be because resistance to metronidazole is very low in Japan (about $5 \%$ ) where health

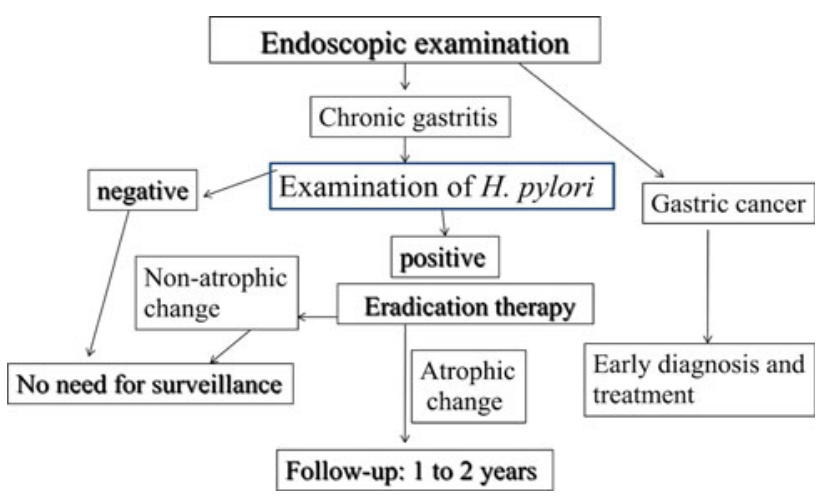

Fig. 4 Strategy for elimination of gastric cancer deaths in Japan 


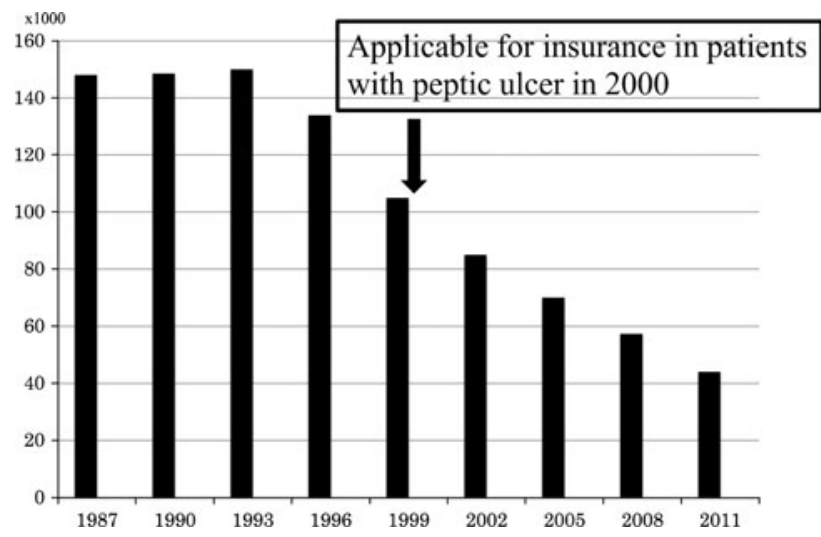

Fig. 5 Changes in incidence of peptic ulcer in Japan

insurance coverage for administration of metronidazole is limited and its use remains uncommon [36]. A recent report indicated that third-line eradication therapy with sitafloxacin was effective for $70 \%$ of the patients in whom secondary eradication therapy was ineffective [47].

Although it is not clear to what extent the use of eradication therapy in patients with $H$. pylori-associated gastritis will inhibit the development of gastric cancer, a good model may be peptic ulcer for which $H$. pylori eradication therapy was first covered by the Japanese national health scheme in 2000. Since then, the incidence of peptic ulcer has decreased dramatically by about $60 \%$ over 10 years (Fig. 5) [48]. In addition, the medical costs of treating ulcers have decreased by no $47 \%$ during that period. Although it is unclear whether the results obtained with gastric cancer will be comparable to those for peptic ulcer, H. pylori eradication therapy (etiologic treatment) for H. pylori-associated gastritis will lead to a long-term decrease of gastric cancer. Such treatment will inhibit the development of peptic ulcer and gastric polyps as well as gastric cancer, suggesting a greater reduction of medical costs than that achieved by providing insurance coverage for $\mathrm{H}$. pylori eradication therapy in patients with peptic ulcer.

There are two potential outcomes of the gastric cancer elimination project suggested here with regard to gastric cancer-related deaths in Japan. One is a definite decrease in the incidence of gastric cancer resulting from the widespread use of $H$. pylori eradication therapy (a direct effect of this therapy). The other is a decrease in the number of deaths resulting from an increase in the diagnosis of early gastric cancer owing to mandatory endoscopy at the time of presentation for chronic gastritis. The target would be to eventually increase the proportion of early gastric cancer from the current $60 \%$ to around $90 \%$, which would make it possible to increase the 5-year survival rate for gastric cancer patients in Japan to approximately $90 \%$. Because the baby boomer generation represents a huge population turning 65 years old and entering the cancer-prone years,

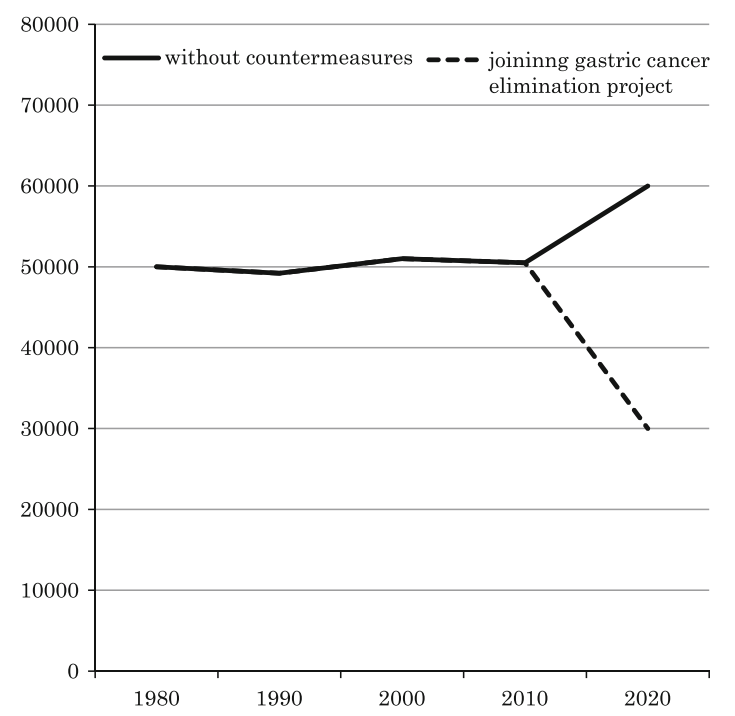

Fig. 6 Anticipation of gastric cancer deaths with or without countermeasures in Japan

the number of deaths from gastric cancer is likely to reach 60,000 in 2020 without any countermeasures. In contrast, if the gastric cancer elimination project is successful and about $50 \%$ of persons with $\mathrm{H}$. pylori infection receive eradication therapy, the number of deaths from gastric cancer will decrease to about 30,000 in 2020 (Fig. 6).

\section{Conclusion}

H. pylori eradication therapy for chronic gastritis achieved the world's first coverage by the Japanese national health insurance scheme in 2013, making a dramatic decrease of gastric cancer-related deaths more realistic. Combining H. pylori eradication therapy with endoscopic surveillance can prevent the development of gastric cancer. Even if gastric cancer develops, most patients are likely to be diagnosed while it is at an early stage, possibly resulting in a large decrease of gastric cancer deaths.

Success with the elimination of gastric cancer in Japan could lead other countries with a high incidence of gastric cancer (China, Korea, and Latin American countries) to consider a similar strategy, suggesting the potential for elimination of gastric cancer around the world.

Conflict of interest Masahiro Asaka belongs to the donation-funded department by Eizai Co. LTD at Hokkaido University Graduate School of Medicine.

Open Access This article is distributed under the terms of the Creative Commons Attribution Noncommercial License which permits any noncommercial use, distribution, and reproduction in any medium, provided the original author(s) and the source are credited. 


\section{References}

1. Warren JR, Marshall BJ. Unidentified curved bacilli on gastric epithelium in active chronic gastritis. Lancet. 1983;1:1273-5.

2. International agency for research on cancer. World Health Organization: schistosomes, liver flukes and Helicobacter pylori. IARC Monogr Eval Carcinog Risk Hum. 1994;61:177-241.

3. Fukase K, Kato M, Kikuchi S, et al. Effect of eradication of Helicobacter pylori on incidence of metachronous gastric carcinoma after endoscopic resection of early gastric cancer: an openlabel, randomised controlled trial. Lancet. 2008;372:392-7.

4. Ferlay J, Shin HR, Bray F, et al. Estimates of worldwide burden of cancer in 2008: GLOBOCAN 2008. Int J Cancer. 2010;127: 2893-917.

5. Marugame T, Matsuda T, Kamo K, et al. Cancer incidence and incidence rates in Japan in 2001 based on the data from 10 population-based cancer registries. Jpn J Clin Oncol. 2007;37:884-91.

6. Nagayo T, Ito M, Yokoyama H, Komgoe T. Early phases of human gastric cancer: morphological study. Gann. 1965;56:101-20.

7. Nakamura K, Sugano H, Takagi K. Carcinoma of the stomach in incipient phase: its histogenesis and histological appearances. Gann. 1968;59:251-8.

8. Kaneko E, Nakamura T, Umeda N, Fujino M, Niwa H. Outcome of gastric carcinoma detected by gastric mass survey in Japan. Gut. 1977;18:626-30.

9. Survival rate in the member hospitals of the association of clinical cancer canters diagnosed in 2000-2004, Cancer statistics in Japan-2012 pp 76-77.

10. Noguchi Y, Yoshikawa T, Tsuburaya A, et al. Is gastric carcinoma different between Japan and the United States? Cancer. 2000;89(11):2237-46.

11. Hundahl SA, Phillips JL, Menck HR. The national cancer data base report on poor survival of US gastric carcinoma patients with gastrectomy. Cancer. 2000;88:921-32.

12. Comparison of 5 year's survival rates between Japan and Western countries. Cancer Statistics in Japan-2006, 2006; pp 59.

13. Schlemper RJ, Itabashi M, Kato Y, et al. Difference in diagnostic criteria for gastric carcinoma between Japanese and Western pathologists. Lancet. 1997;349:1725-9.

14. Schlemper RJ, Riddell RH, Kato Y, et al. The Vienna classification of gastrointestinal epithelial neoplasia. Gut. 2000;47(2): 251-5.

15. Yamada H, Ikegami M, Shimoda T, et al. Long-term follow-up study of gastric adenoma/dysplasia. Endoscopy. 2004;36:390-6.

16. Park SY, Jeon SW, Jung MK, et al. Long-term follow-up study of gastric intraepithelial neoplasias: progression from low-grade dysplasia to invasive carcinoma. Eur J Gastroenterol Hetatol. 2008;20:966-70.

17. Dinis-Ribeiro M, Areia M, De Vries AC, et al. Management of precancerous conditions and lesions in the stomach (MAPS): guideline from the European Society of Gastrointestinal Endoscopy (ESGE), European Helicobacter Study Group (EHSG), European Society of Pathology (ESP), and the Sociedade Portuguesa de Endoscopia Digestiva (SPED). Endoscopy. 2012;44:74-94.

18. Dixon MF. Gastrointestinal epithelial neoplasia: Vienna revised. Gut. 2002;51:130-1.

19. Miehlke S, Kirsch C, Dragosics B, et al. Helicobacter pylori and gastric cancer: current status of the Austrian Czech German gastric cancer prevention trial (PRISMA Study). World J Gastroenterol. 2001;7:243-7.

20. Wong BC, Lam SK, Wong WM, et al. Helicobacter pylori eradication to prevent gastric cancer in a high-risk region of China: a randomized controlled trial. JAMA. 2004;291:187-94.
21. Asaka M, Kimura T, Kato M, et al. Possible role of Helicobacter pylori infection in early gastric cancer development. Cancer. 1994;73:2691-4.

22. Whiting JL, Sigurdsson A, Rowlands DC, et al. The long term results of endoscopic surveillance of premalignant gastric lesions. Gut. 2002;50:378-81.

23. Uemura N, Okamoto S, Yamamoto S, et al. Helicobacter pylori infection and the development of gastric cancer. N Engl J Med. 2001;345:784-9.

24. Arima N, Adachi K, Katsube T, et al. Predictive factors for metachronous recurrence of early gastric cancer after endoscopic treatment. J Clin Gastroenterol. 1999;29:44-7.

25. Nasu J, Doi T, Endo H, Nishina T, et al. Characteristics of metachronous multiple early gastric cancers after endoscopic mucosal resection. Endoscopy. 2005;37:990-3.

26. $\mathrm{Wu} \mathrm{CY}$, Kuo KN, Wu MS, et al. Early Helicobacter pylori eradication decreases risk of gastric cancer in patients with peptic ulcer disease. Gastroenterology. 2009;137(5):1641-9.

27. You WC, Brown LM, Zhang L, et al. Randomized double-blind factorial trial of three treatments to reduce the prevalence of precancerous gastric lesions. J Natl Cancer Inst. 2006;98:974-83.

28. Ma JL, Zhang L, Linda M, et al. Fifteen-year effects of Helicobacter pylori, garlic, and vitamin treatments on gastric cancer incidence and mortality. J Natl Cancer. 2012;104:488-92.

29. Maehata Y, Nakamura S, Fujisawa K, et al. Long-term follow-up study about preventive effect of $H$. pylori eradication for the incidence of metachronous gastric cancer after endoscopic resection of early gastric cancer. Gastrointest Endosc. 2012;75:39-46.

30. Kato M, Asaka M, Kikuchi S. Long-term follow-up study about preventive effect of $H$. pylori eradication for the Incidence of metachronous gastric cancer after endoscopic resection of primary early gastric cancer. Gastroenterology. 2012;142(5 Supple1):S3.

31. Harvard Report on Cancer Prevention. Volume 1: causes of human cancer. Cancer Causes Control. 1996;7(Suppl 1):S3-59.

32. Olsen JH, Andersen A, Dreyer L, et al. Summary of avoidable cancers in the Nordic countries. APMIS Suppl. 1997;76:141-6.

33. Number of incidence by cancer site 2007. Cancer statistics in Japan-2012, Tokyo, Japan 2012; pp 15.

34. Tsukuma H, Tanaka H, Ajiki W, Oshima A. Liver cancer and its prevention. Asian Pac J Cancer Prev. 2005;6:244-50.

35. Makuuchi M, Kokudo N, Arii S, et al. Development of evidencebased clinical guidelines for the diagnosis and treatment of hepatocellular carcinoma in Japan. Hepatol Res. 2008;38:37-51.

36. Trends in site-specific crude mortality rate 1965-2010. Cancer Statistics in Japan-2011, Tokyo, Japan, 2011; pp 26.

37. Asaka M, Kato M, Takahashi S, et al. Guidelines for the management of Helicobacter pylori infection in Japan: 2009 revised edition. Helicobacter. 2010;15:1-20.

38. Blaser MJ. Gastric Campylobacter-like organism, gastritis, and peptic ulcer diseases. Gastroenterology. 1987;93:371-83.

39. Keates S, Hitti YS, Upton M, et al. Helicobacter pylori infection activates NF- $\mathrm{KB}$ in gastric epithelial cells. Gastroenterology. 1997;113:1099-109.

40. Asaka M, Sugiyama T, Nobuta A, et al. Atrophic gastritis and intestinal metaplasia in Japan: results of a large multicenter study. Helicobacter. 2001;6:294-9.

41. Correa P. Human gastric carcinogenesis: a multistep and multifactorial process. Cancer Res. 1992;52:6735-40.

42. Asaka M, Takeda H, Sugiyama T, et al. What role does Helicobacter pylori play in gastric cancer? Gastroenterology. 1997;113:S56-60.

43. Asaka M. A new approach for elimination of gastric cancer in Japan. Int J Cancer. 2013;132:1272-6. 
44. Kodama M, Murakami K, Okimoto T, et al. Ten-year prospective follow-up of histological changes at five points on the gastric mucosa as recommended by the updated Sydney system after Helicobacter pylori eradication. J Gastroenterol. 2012;47: 394-403.

45. Asaka M, Kato M, Graham DY. Strategy for eliminating gastric cancer in Japan. Helicobacter. 2010;15:486-90.

46. Akamatsu $\mathrm{T}$, Ichikawa $\mathrm{S}$, Okudaira $\mathrm{S}$, et al. Introduction of an examination and treatment for Helicobacter pylori infection in high school health screening. J Gastroenterol. 2011;46:1353-60.
47. Murakami K, Furuta T, Ando T et al Multi-center randomized controlled study to establish the standard third-line regimen for Helicobacter pylori eradication in Japan. J Gastroenterol. 2013; 48 published online.

48. Patient survey 2011, Statistics and Information Department, Ministry of Health, Labour and welfare, 2011; pp 53. 